\title{
Aeroacoustic Assessment of Conceptual Low-Noise High-Lift Wing Configurations
}

\author{
Hua-Dong Yao $^{1 *}$, Lars-Erik Eriksson $^{1 \dagger}$, Lars Davidson $^{1 \ddagger}$ \\ Olof Grundestam $^{2 \S}$, Shia-Hui Peng ${ }^{1,2}$ \& Peter E. Eliasson ${ }^{2 \|}$ \\ 1. Chalmers University of Technology, SE-412 96 Gothenburg, Sweden \\ 2. Swedish Defence Research Agency (FOI), SE-164 90 Stockholm, Sweden
}

\begin{abstract}
Aeroacoustics performance is assessed for three conceptual low-noise and high-lift wings using the surface integral methods of acoustic analogy, including the Kirchhoff method, the Ffowcs Williams and Hawkings method and the Curle method. A new way is proposed to define the integral surface that encloses the core flow region for the first two methods on the basis of the vorticity magnitudes. Both of the first two methods are used to compute the noise generated by the core region of the flow, which is responsible for most of the noise generation. The results obtained by the FWH method support those given by the Kirchhoff method. The Curle analogy approach is adopted to calculate the noise by the boundary layer flow attached on the walls inside the core flow region. Through comparison of the results of the three approaches, the levels of the contribution to the noise by the volume flow and the boundary layers are studied for the configurations. Finally, the mechanism of efficiency on the noise reduction is presented for the three configurations.
\end{abstract}

\section{Introduction}

Noise reduction is a key issue in conceptual designs of silent aircraft, for which numerical simulations have been increasingly employed in aeroacoustic analysis with the rapid development of computing facilities. Nonetheless, with the limits of computational costs in engineering applications, computational aeroacoustics (CAA) is challenged for its accuracy in predicting the far-field noise, where the sound wave in long distance transmission is often characterized as fluctuations with small magnitudes.

Instead of direct computation of the noise, hybrid methods for acoustic analogy have been widely used for predictions of the far-field noise induced by the aerodynamic flow. A hybrid method typically includes two steps. The near-field flow is computed first using computational fluid dynamics (CFD) techniques, from which flow-generated noise sources are extracted. The flow properties are then formulated in relevant forms as source terms in an acoustic analogy, by which the far-field noise level is estimated.

High-lift flows are almost always turbulent, which can be handled in a hierarchical way of different simulation approaches in CFD, ranging from direct numerical simulation (DNS) and large eddy simulation (LES) to Reynolds-averaged Navier-Stokes (RANS) methods. At very high Re numbers - the usual case in industry - it is recognized that the cost of using DNS and LES is too high. On the other hand, conventional RANS approaches provide only mean flow information but no instantaneous flow fluctuation that is required in aeroacoustic analysis, although it is apparently beneficial to save computation costs. However, the recent development of hybrid RANS-LES method, which uses turbulence-resolved modeling approaches, has made

\footnotetext{
*Department of Applied Mechanics, huadong@chalmers.se

$\dagger$ Department of Applied Mechanics, lars-erik.eriksson@chalmers.se

${ }^{\ddagger}$ Department of Applied Mechanics, lada@chalmers.se

$\S$ Department of Aeronautics and Systems Technology, olof.grundestam@foi.se

『Department of Aeronautics and Systems Technology, shia-hui.peng@foi.se

\| Department of Aeronautics and Systems Technology, peter.eliasson@foi.se

Copyright (C) 2012 by the American Institute of Aeronautics and Astronautics, Inc. The U.S. Government has a royalty-free license to exercise all rights under the copyright claimed herein for Governmental purposes. All other rights are reserved by the copyright owner.
} 
it possible to capture turbulent sources of the noise at low cost. This is the approach adopted in the present work.

The surface integral methods of acoustic analogy include the Kirchhoff method, ${ }^{1-3}$ the Ffowcs Williams and Hawkings (FWH) method ${ }^{4}$ and the boundary element method (BEM). ${ }^{5}$ Theoretical and numerical comparisons of the first two methods have been made in CAA analysis of rotor noise ${ }^{6-9}$ and jet noise. ${ }^{10,11}$ In addition to these methods, the vortex sound theory has also recently been applied in numerical predictions of the noise generated by a 3D profile of the high-lift wing. ${ }^{12,13}$ Along with the analogy methods referred to above, the noise induced by the turbulent boundary layer to a stationary wall can be studied using the Curle method. ${ }^{14-16}$

The major objective of this paper is to assess aeroacoustic performance for three conceptual configurations with low noise and high-lift using the Kirchhoff method, the FWH method and the Curle method. Comparative analyses are made of far-field noise levels in order to evaluate the efficiency in noise reduction. The potential of noise reduction for the high-lift devices evaluated is highlighted. In a separate paper, a detailed study of the three acoustic analogy methods will be further reported. ${ }^{17}$

\section{Surface Integral Methodologies}

\section{II.A. The Kirchhoff Method}

The Kirchhoff method is based on the requirement that the integral surface is located in the flow region where the wave propagation condition is fulfilled.As derived by Lyrintzis, ${ }^{2}$ the form of the Kirchhoff equation is written as:

$$
\begin{aligned}
p_{\text {total }, K}^{\prime}(\vec{x}, t)= & \frac{1}{4 \pi} \int_{f=0}\left\{\frac{\cos \theta}{R^{2}}\left[p^{\prime}(\vec{y}, \tau)\right]_{r e t}-\frac{1}{R}\left[\frac{\partial p^{\prime}(\vec{y}, \tau)}{\partial n}\right]_{r e t}\right. \\
& \left.+\frac{\cos \theta}{c_{0} R}\left[\frac{\partial p^{\prime}(\vec{y}, \tau)}{\partial \tau}\right]_{\text {ret }}\right\} d S
\end{aligned}
$$

where $R$ is the modulus of $\vec{r}, \vec{r}=\vec{x}-\vec{y}, \vec{n}$ is the vector normal to the integral surface, $\cos \theta=\left(r_{i} / R\right) n_{i}$, $c_{0}$ is the speed of sound and $f(\vec{y})=0$ denotes the integral surface which encloses the noise source of the flow. Moreover, the terms in brackets marked by the subscript 'ret' are evaluated at the retarded time $\tau=t-R / c_{0}$.

\section{II.B. The FWH Method for a Permeable Surface}

The original form of the FWH equation ${ }^{18}$ indicates that it can be applied to a stationary permeable surface instead of a rigid wall. 7,8 It reads:

$$
\begin{aligned}
p_{\text {total }, F}^{\prime}(\vec{x}, t)= & -\frac{1}{4 \pi} \frac{\partial}{\partial x_{i}} \int_{f=0}\left\{\frac{1}{R}\left[p^{\prime} \delta_{i j}-\tau_{i j}+\rho u_{i} u_{j}\right]_{r e t} n_{j}\right\} d S \\
& +\frac{1}{4 \pi} \frac{\partial}{\partial t} \int_{f=0}\left\{\frac{1}{R}\left[\rho u_{j} n_{j}\right]_{r e t}\right\} d S
\end{aligned}
$$

where $\tau_{i j}$ is the viscous and modeled turbulent stress tensor, which is neglected in the present analysis. By assuming that the permeable surface encloses the core flow region, the volume integral term for quadrupole source in Eq. (2) has been neglected.

\section{II.C. The Curle Method}

If stationary rigid walls are present in a flow, the noise is predicted by the Curle equation ${ }^{14}$ as:

$$
p_{\text {total }, C}^{\prime}(\vec{x}, t)=-\frac{1}{4 \pi} \frac{\partial}{\partial x_{i}} \int_{f=0}\left\{\frac{1}{R}\left[p^{\prime}\right]_{r e t} n_{i}\right\} d S+\frac{1}{4 \pi} \frac{\partial}{\partial x_{i} \partial x_{j}} \int_{V}\left\{\frac{1}{R}\left[T_{i j}\right]_{r e t}\right\} d V
$$

The first integral term in Eq (3) depicts the noise induced by the boundary layer flow over rigid walls. Our interest in the present analysis is the noise from boundary layers. Thus, the volume integral term of the Lighthill stress tensor is neglected, in which $T_{i j}=\left(p^{\prime}-\rho^{\prime} c_{o}^{2}\right) \delta_{i j}-\tau_{i j}+\rho u_{i} u_{j}$. 


\section{Numerical Simulation Settings}

Three high-lift configurations, ${ }^{19}$ termed Config. 1, Config. 2 and Config. 4, were assessed in this paper. The baseline configuration is set as Config. 1. Figure 1 shows the components of the high-lift devices for each configuration. The baseline configuration consists of a double slotted part-span flap. The second one adopts a structure with a single slotted part-span flap. The last one uses the same flap as the second one, except for the Krueger flap. A cut plane for each configuration is illustrated in Fig. 2.

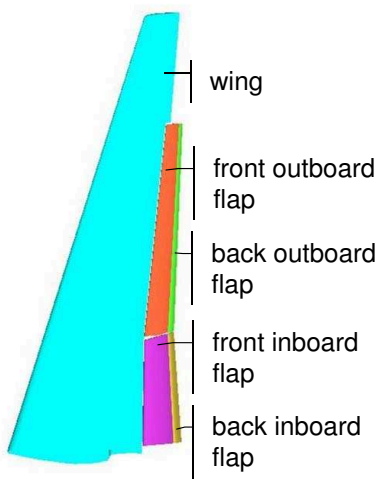

(a) Config. 1 (baseline)

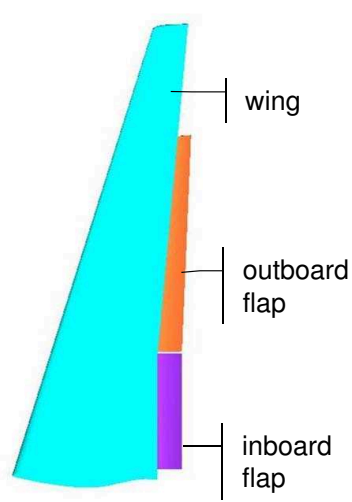

(b) Config. 2

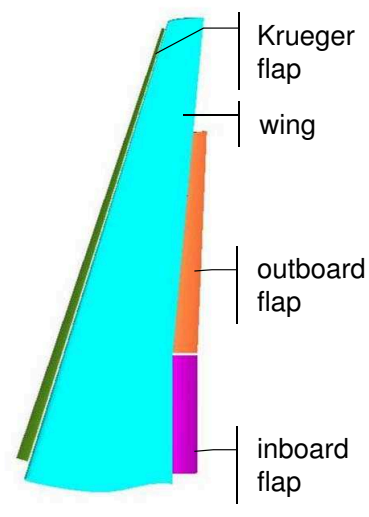

(c) Config. 4

Figure 1. The components of the three configurations.

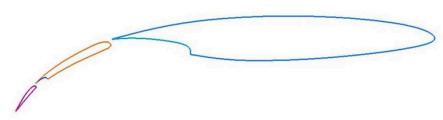

(a) Config. 1 (baseline)

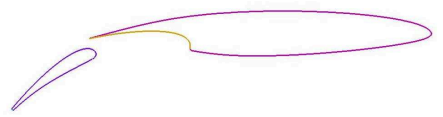

(b) Config. 2

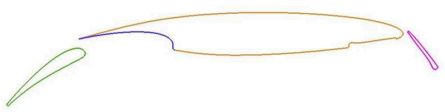

(c) Config. 4

Figure 2. Cut planes at mid-span of the three configurations.

\section{III.A. Flow Simulation}

The flow fields of the three configurations are computed under the same flow conditions using a hybrid RANS-LES model. The freestream Mach number is 0.2 , and the Reynolds number is $1.7 \times 10^{7}$. The first two configurations operate at the stall angle while the last one keeps the same angle of attack as Config. 2. The configurations are attached to a common fuselage body in the numerical simulations. The 3D geometry of Config. 1 is shown in Fig. 3. The symmetrical boundary condition is applied at the symmetry plane of the computational domain, which corresponds to the symmetry plane at the fuselage.

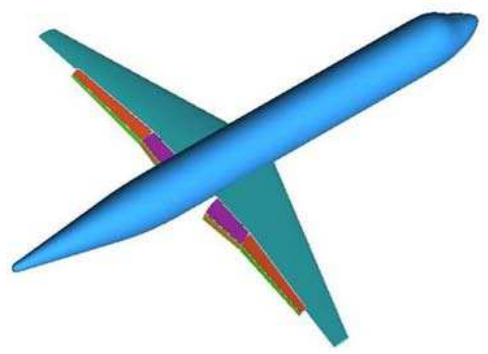

Figure 3. The sketch of Config. 1 that is used. Because of symmetry, the simulations are carried out for half of the configuration. 


\section{III.B. Aeroacoustics Calculation}

When using the surface integral methods of acoustic analogies, a integral surface needs to be defined for integration. According to the preconditions for applying the Kirchhoff method and the permeable surface form of the FWH method, the core region of the flow field must be enclosed within the integral surface because it is responsible for most of noise generation.$^{9,10}$ A common integral surface is employed in the present calculations using the Kirchhoff method and the FWH method, although the restriction on the location of the surface is weaker for the latter method.

The core flow region can be evaluated through vorticity because it characterizes the feature of turbulent flow structures. Therefore, in order to make the integral surface enclose the core region in the Kirchhoff method and the FWH method, the location of the surface can be determined on the basis of vorticity magnitudes, which marks the strength of the flow structures. A rough estimation of vorticity is made in a precursor RANS computation. As an example, the integral surface defined for Config. 1 is shown in Fig. 4. The integral surface is marked with six colors that indicate the partition strategy in the surface generation. The end of the surface in the downstream direction is kept open so as to reduce the artificial noise that is caused by vortices crossing the surface.

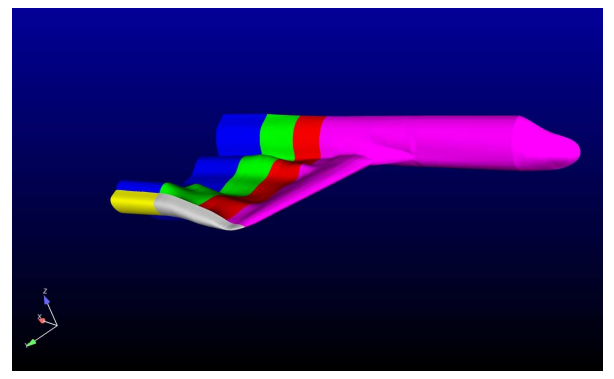

Figure 4. The integral surface that is defined for Config. 1 in the Kirchhoff method and the FWH method.

In addition to evaluating the noise from the core flow region by the above two methods, the contribution from the boundary layer is calculated by the Curle method. When using this method, the integral surface is set onto the rigid walls.

Observers in the far field are placed in a circle in the symmetry plane of the computational domain with respect to a reference position in the core flow region. The reference position is located at the root of the main wing. The distance between an arbitrary observer and the reference position is $15 L$, where $L$ is the wing span length. To decide the directivity of the noise level, the polar axis for this placement strategy points to the upstream direction.

\section{Numerical Results}

\section{IV.A. Calculate Noise by the Kirchhoff Method}

Noise contributed by the core flow region is calculated with the Kirchhoff method for the three configurations in this section. Quantities relative to the fluctuating pressure, which are obtained from the hybrid method of RANS and LES, are integrated over the integral surface to compute the noise using Eq. (1). The sound pressure level (SPL) is used to analyze the noise as $S P L=20 \log \left(p / p_{\text {ref }}\right)$. Here $p_{\text {ref }}$ depicts the reference pressure. Figure 5 shows SPL of $p_{\text {total }, K}^{\prime}$ at an observer at $270^{\circ}$ for the three configurations. The observer is evaluated because its location is vertically below the airframe. The actual amplitudes of the figures in this paper are removed for confidentiality. It is found that a highest SPL for Config. 1 is observed at lower Strouhal numbers, which is defined as $S t=f L / U$, where $L$ is the characteristic length of the chord of the wing and $U$ is the mean flow velocity. However, discrepancies in the amplitude levels decrease in the range of $S t$ higher than 2. The overall sound pressure level (OASPL) of the noise is shown in Fig. 6. The polar axis for the OASPL points to the upstream of the mean flow. Subfigure (a) displays the OASPL for the total noise which includes all resolved frequencies, and subfigure (b) displays the OASPL for the noise of frequencies higher than $20 \mathrm{~Hz}$. It is observed in subfigure (a) that the total noise level of Config. 4 is lower than the other two from 6 to $10 \mathrm{~dB}$. However, it is found in subfigure (b) that the noise of frequencies higher than $20 \mathrm{~Hz}$ stays at the same level for the three configurations. 


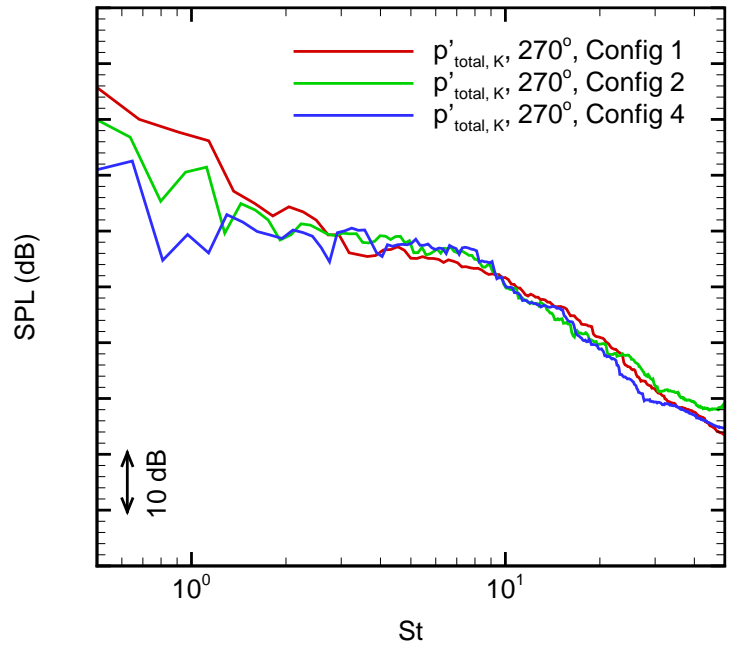

Figure 5. SPL of $p_{\text {total }, K}^{\prime}$ at observer $270^{\circ}$. The Kirchhoff method.

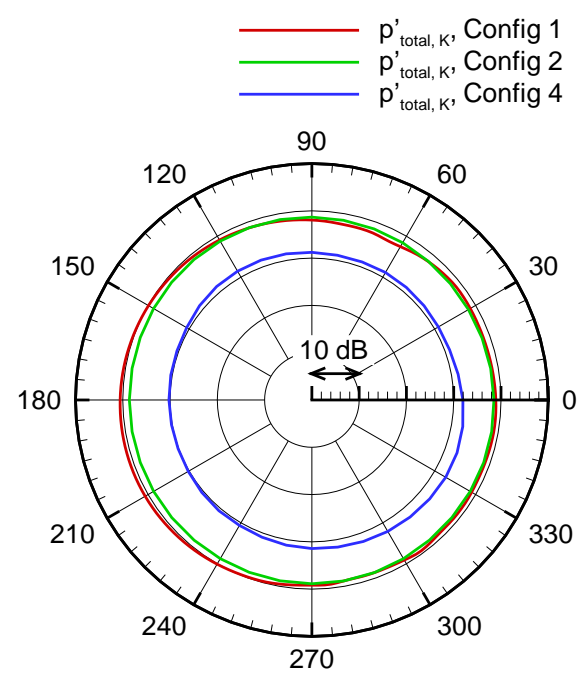

(a) All resolved frequencies.

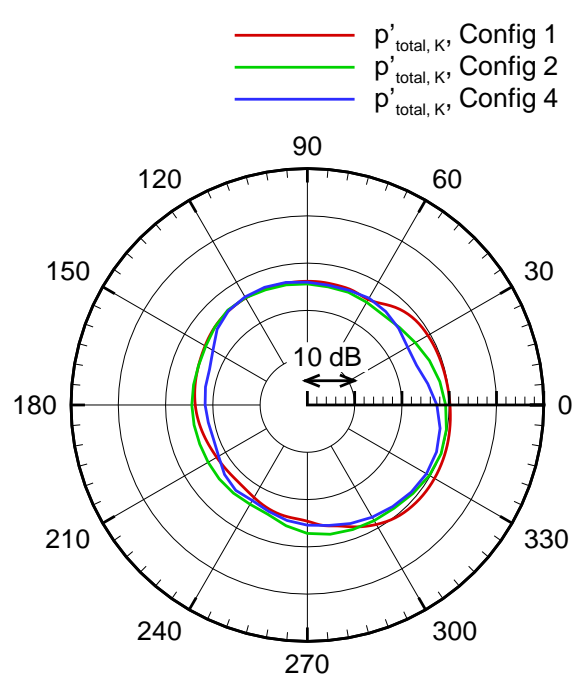

(b) Resolved frequencies higher than $20 H z$.

Figure 6. OASPL of $p_{\text {total }, K}^{\prime}$. The Kirchhoff method. 


\section{IV.B. Calculate Noise by the FWH Method}

In addition to the Kirchhoff method, the FWH method is adopted to calculate the noise from the core flow region according to Eq. (2). The SPL of $p_{\text {total }, F}^{\prime}$ for the configurations at observer $270^{\circ}$ are compared in Fig. 7. It is observed that the energy level of the noise for Config. 1 is in general highest. Subfigure (a) in Fig. 8 shows the OASPL of the total noise involving energy over all resolved frequencies, and subfigure (b) shows the OASPL for the noise of frequencies higher than $20 \mathrm{~Hz}$. It is observed in subfigure (a) that the noise level is significantly reduced for Config. 4 compared to the other two configurations. The noise level at high frequencies $(>20 \mathrm{~Hz})$ in subfigure (b) also shows the reduction effects based on Config. 1. Furthermore, by comparing the two figures, it is found that the noise at low frequencies $(<20 H z)$ is not dominant in Config. 4. A sudden energy bulk of OASPL is observed in the upstream direction for Config. 1. We assume that it is caused by the unsuitable location of the integral surface, which is too close to rigid walls, as well as the mesh density distribution inside the surface.

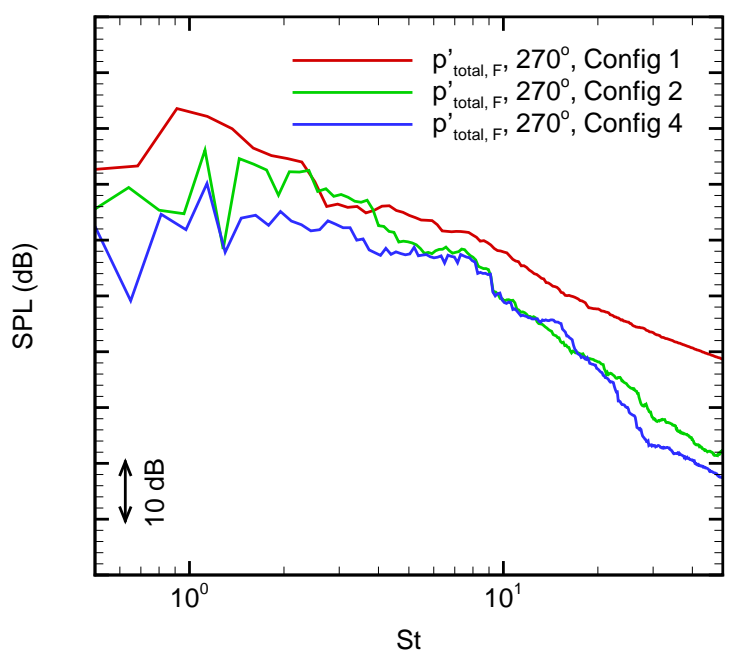

Figure 7. SPL of $p_{\text {total, } F}^{\prime}$ at observer $270^{\circ}$. The FWH method.

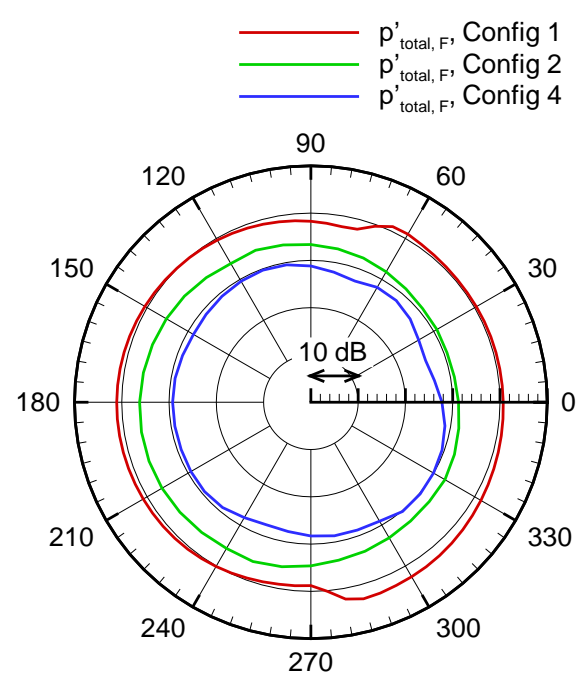

(a) All resolved frequencies.

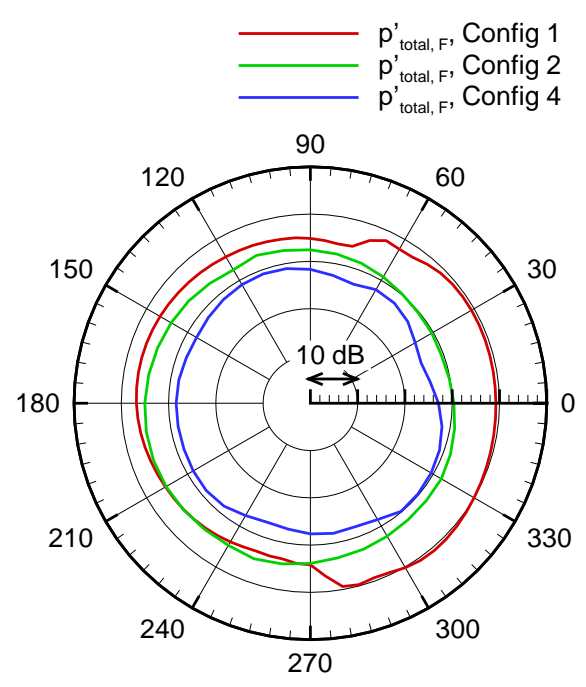

(b) Resolved frequencies higher than $20 H z$.

Figure 8. OASPL of $p_{\text {total }, F}^{\prime}$. The FWH method. 


\section{IV.C. Calculate Noise from the Boundary Layer by the Curle Method}

Noise contributed by the volume flow and the boundary layer flow is computed together when using the Kirchhoff method and the FWH method. However, the contribution by the boundary layer flow can be studied specifically by the Curle method if the volume integral term is neglected in Eq. (3). The SPL of $p_{\text {total }, C}^{\prime}$ at $270^{\circ}$ is presented in Fig. 9. The results indicate that the noise induced by the turbulent boundary layer of Config. 2 is the lowest one. The OASPL of Config. 2 in Fig. 10 (a) and (b) are lowest in the downward directions. A comparison of the two subfigures indicates that the percentage of the noise of the boundary layer flow in frequencies lower than $20 \mathrm{~Hz}$ is not high.

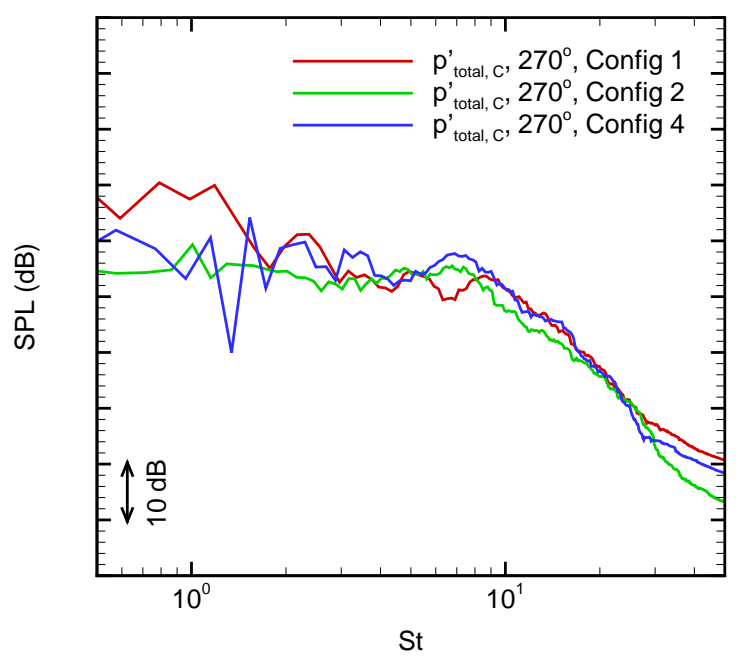

Figure 9. SPL of $p_{\text {total, } C}^{\prime}$ at observer $270^{\circ}$. The Curle method

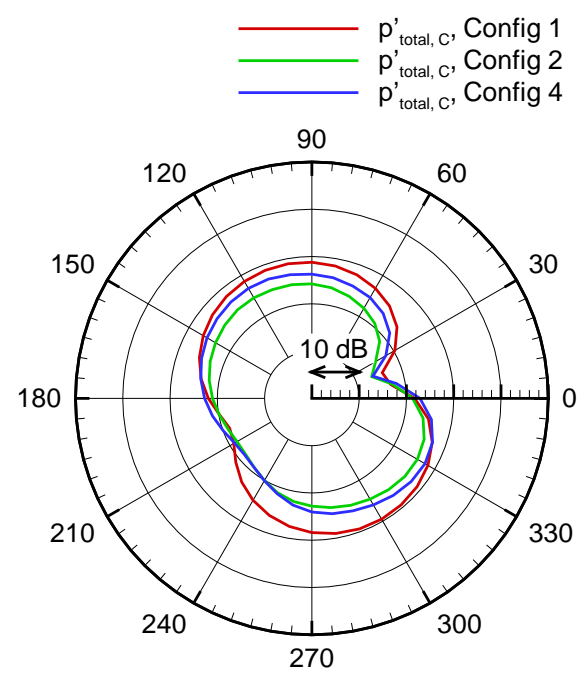

(a) All resolved frequencies.

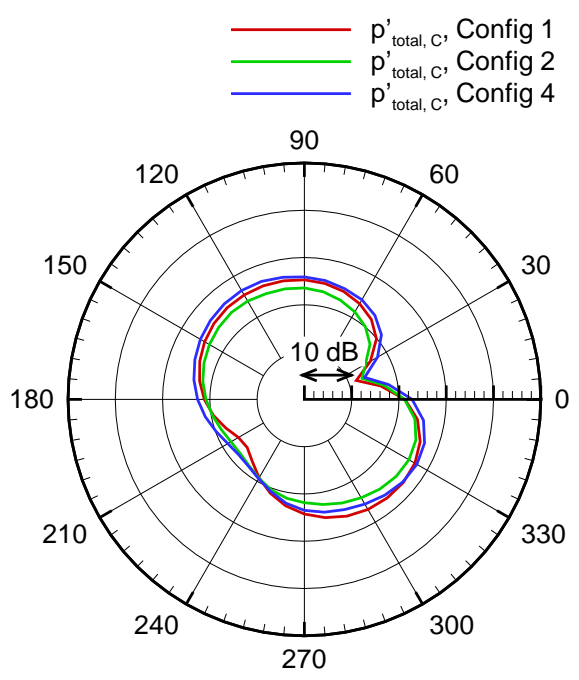

(b) Resolved frequencies higher than $20 H z$.

Figure 10. OASPL of $p_{t o t a l, C}^{\prime}$. The Curle method.

\section{IV.D. Overall Aeroacoustic Assessment on Three Configurations}

To assess the configurations quantitatively, Table 1 lists the comparative magnitudes of the SPL, in which the results of Config. 1 are set to zero as the references. It is seen that the noise reduction of the last two configurations decreases with increasing $S t$ number. The low frequency noise, $S t=1$, is reduced significantly 
in Config. 4. The effect appears not only for the total noise computed by the Kirchhoff method and the FWH method, but also for the noise from the boundary layer that is computed by the Curle method. The magnitudes of the OASPL for the noise over the resolved frequency band in the downward directions are shown in the same manner in Table 2. It is found that the total noise from the core flow region is brought down considerably for Config. 4. However, the reduction efficiency of Config. 4 on the boundary layer noise decreases slightly as compared with Config. 2 in those directions. This implies that the volume flow is the dominant noise source for the high-lift devices which operate at stall angle. That is, the noise reduction efficiency for the last two configurations depends on their capabilities to control the unsteady volume flow.

Table 3 lists magnitudes of the OASPL in three downward directions for the noise omitting energy in frequencies lower than $20 \mathrm{~Hz}$. The results given by the Kirchhoff method are similar for all configurations. However, those given by the FWH method show apparent changes, and Config. 4 reduces the noise. Here we prefer to trust the FWH method because it takes into account the non-linear effect in flow. It is seen that a sharp depression appears at the observer $300^{\circ}$ for Config. 2 and Config. 4 using the FWH method. However, as discussed in Fig. 8, there is an energy bulk in the upstream directions which is probably caused by too coarse a mesh. Comparing with the OASPL magnitudes in Table 2, the extent of the reduction in the last two configurations in Table 3 generally becomes much lower if low frequency noise is omitted. This therefore suggests that the noise in the low frequency range is important.

Table 1. Difference in SPL (dB) relative to Config. 1 at Observer $270^{\circ}$.

\begin{tabular}{l|c|c|c|c|c|c|c|c|c}
\hline & \multicolumn{3}{|c|}{ Kirchhoff method } & \multicolumn{3}{c|}{ FWH method } & \multicolumn{3}{c}{ Curle method } \\
\cline { 2 - 10 } & $S t=1$ & St=5 & $S t=10$ & $S t=1$ & $S t=5$ & $S t=10$ & $S t=1$ & $S t=5$ & $S t=10$ \\
\hline Config. 1 & 0 & 0 & 0 & 0 & 0 & 0 & 0 & 0 & 0 \\
\hline Config. 2 & -6.26 & 2.41 & -1.67 & -15.28 & -4.81 & -8.60 & -9.04 & 1.39 & -4.49 \\
\hline Config. 4 & -18.29 & 2.63 & -1.42 & -19.74 & -6.97 & -8.89 & -12.70 & -1.43 & -0.06 \\
\hline
\end{tabular}

${ }^{*}$ The values of Config. 1 are set to 0 and are taken as references for those of the other two configurations.

Table 2. OASPL (dB) magnitudes at three observers.

\begin{tabular}{c|c|c|c|c|c|c|c|c|c}
\hline & \multicolumn{3}{|c|}{ Kirchhoff method } & \multicolumn{3}{c|}{ FWH method } & \multicolumn{3}{c}{ Curle method } \\
\cline { 2 - 10 } & $240^{\circ}$ & $270^{\circ}$ & $300^{\circ}$ & $240^{\circ}$ & $270^{\circ}$ & $300^{\circ}$ & $240^{\circ}$ & $270^{\circ}$ & $300^{\circ}$ \\
\hline Config. 1 & 0 & 0 & 0 & 0 & 0 & 0 & 0 & 0 & 0 \\
\hline Config. 2 & -1.34 & -0.30 & -0.33 & -4.41 & -4.17 & -9.50 & -4.82 & -5.58 & -4.90 \\
\hline Config. 4 & -9.46 & -7.75 & -6.99 & -11.94 & -10.50 & -13.56 & -4.78 & -4.35 & -3.48 \\
\hline
\end{tabular}

$\dagger$ The values of Config. 1 are set to 0 and are taken as references for those of the other two configurations.

Table 3. OASPL (dB) magnitudes at three observers, disregarding the noise in low frequencies $(f<20 \mathrm{~Hz})$.

\begin{tabular}{l|c|c|c|c|c|c|c|c|c}
\hline & \multicolumn{2}{|c|}{ Kirchhoff method } & \multicolumn{3}{c|}{ FWH method } & \multicolumn{3}{c}{ Curle method } \\
\cline { 2 - 10 } & $240^{\circ}$ & $270^{\circ}$ & $300^{\circ}$ & $240^{\circ}$ & $270^{\circ}$ & $300^{\circ}$ & $240^{\circ}$ & $270^{\circ}$ & $300^{\circ}$ \\
\hline Config. 1 & 0 & 0 & 0 & 0 & 0 & 0 & 0 & 0 & 0 \\
\hline Config. 2 & 1.67 & 2.59 & -0.93 & 0.84 & -0.29 & -7.68 & 0.11 & -2.40 & -2.85 \\
\hline Config. 4 & 0.74 & 0.84 & -1.77 & -6.44 & -6.54 & -11.75 & 0.22 & -0.77 & -1.31 \\
\hline
\end{tabular}

$\dagger$ The values of Config. 1 are set to 0 and are taken as references for those of the other two configurations.

It is also interesting to study the acoustic performance of the common components of the high-lift wing. As indicated by Eq. (3), the noise from the boundary layer flow on the rigid wall can be calculated individually. This advantage is utilized here to study the noise related to a certain rigid wall. Because the wing is common 
to the three configurations, it is reasonable to compare its noise contribution within the configurations using the Curle method. The main wing is split into the wing part and the cove part. Furthermore, the noise contribution from the flaps can be evaluated as two groups, that is, the outboard flap group and the inboard flap group, because Config. 1 is attached by two flaps both at the inboard and outboard parts of the main wing.

The noise from the wing part is shown in Fig. 11. As displayed in subfigure (a) of Fig. 11, the SPL of Config. 1 is highest at frequencies lower than $S t=1.3$. Subfigure (b) shows that the OASPL of Config. 1 is the highest one. The other two configurations have equal noise levels. Noise from the cove part is shown in Fig. 12. As shown in subfigure (a), the SPL of Config. 1 is lowest at frequencies ranging from $S t=3$ to 25. Subfigure (b) shows that the OASPL of Config. 1 is the lowest one. However, the cove parts of the other two configurations have equal noise levels. This phenomenon is also observed for the wing part. Figure 13 displays the noise from the outboard flap group, where (b) indicates that the OASPL of Config. 4 is the highest one, although Config. 1 has two flaps. It is shown in Fig. 14 that the noise is induced by the inboard flap group. Subfigure (b) indicates that the OASPL of the three configurations stay in the same level. Figure 15 shows the noise from the Krueger flap of Config. 4. Taking the total noise over all walls as reference, its noise contribution is not significant.

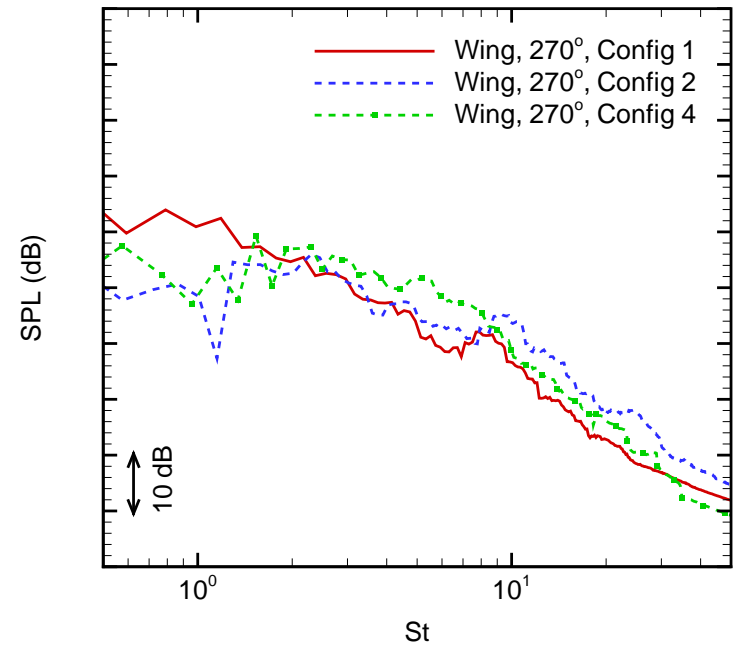

(a) SPL at $270^{\circ}$.

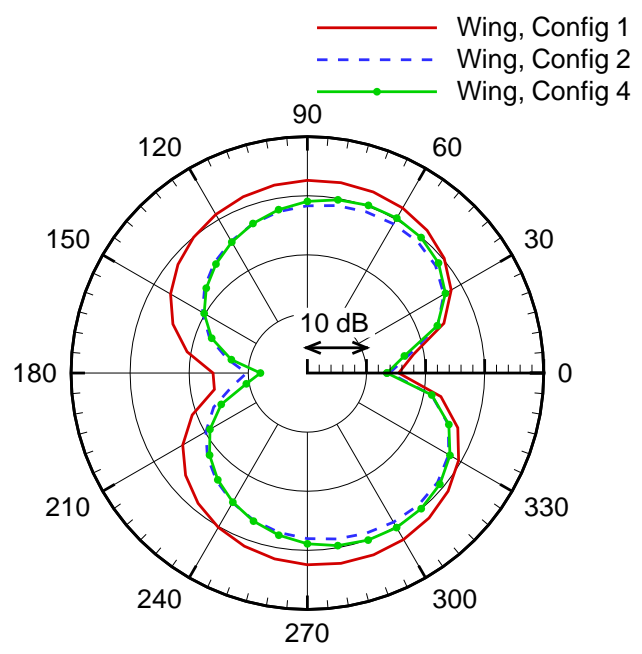

(b) OASPL.

Figure 11. Comparison of the three configurations with SPL and OASPL of the noise, which is induced by the boundary layer on the wing part of the main wing.

\section{Conclusion}

Aeroacoustic performance is assessed for three configurations that are 3D low-noise high-lift wings. Three surface integral methods of acoustic analogies are adopted in the assessment, including the Kirchhoff method, the FWH method and the Curle method. The first two methods are used to study the total noise generated by the core region of the flow. The integral surface to enclose the region is defined on the basis of preliminary RANS results of vorticity. The noise generated by the turbulent boundary layers is then studied with the Curle method.

The reduction efficiency of Config. 4 to the total noise is better than Config. 2, if Config. 1 is taken as baseline. Considering the core region that involves the volume flow and the boundary layers, the first type of flow represents the dominant noise source for the high-lift devices at stall angle when comparing the OASPL between the Curle method and the other two methods. This means that the noise reduction efficiency of the high-lift wing depends on its capability to depress the unsteady volume flow. When the noise lower than 20 $\mathrm{Hz}$ is omitted, the reduction level in the OASPL does not decrease as quickly as that of the noise over all resolved frequencies. Thus, the noise in the low frequency range is depressed to a great extent by the last two configurations. 


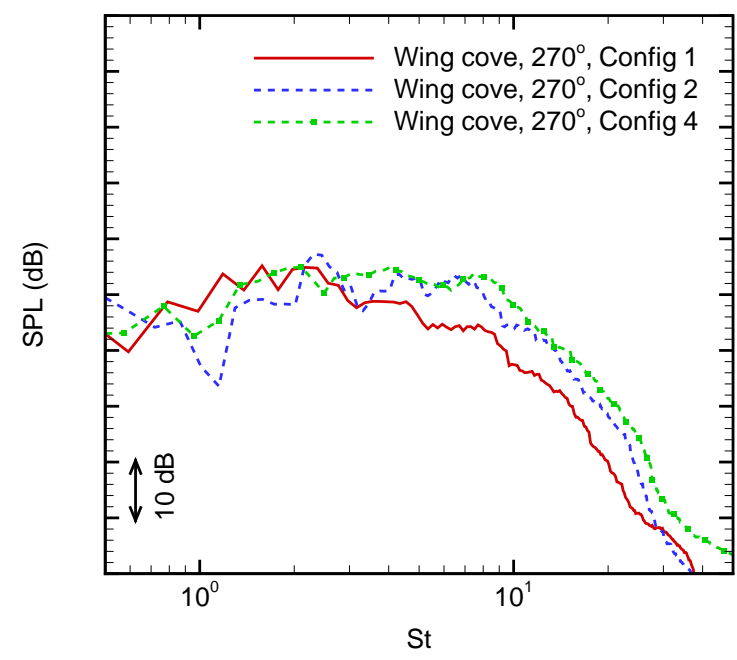

(a) SPL at $270^{\circ}$.

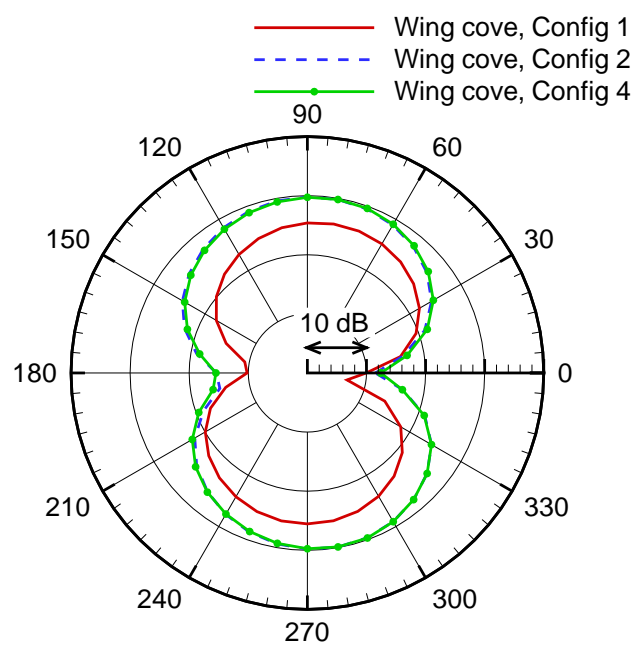

(b) OASPL.

Figure 12. Comparison of the three configurations with SPL and OASPL of the noise, which is induced by the boundary layer on the cove part of the main wing.

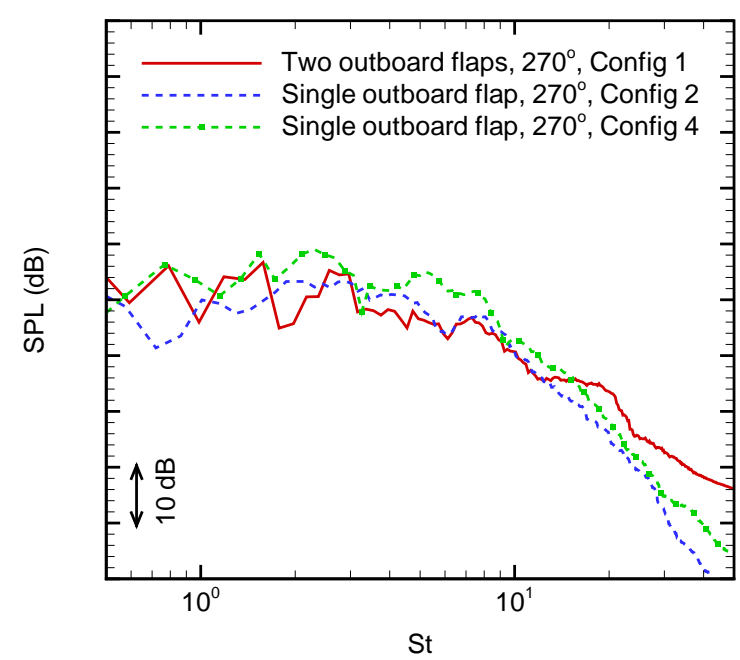

(a) SPL at $270^{\circ}$.

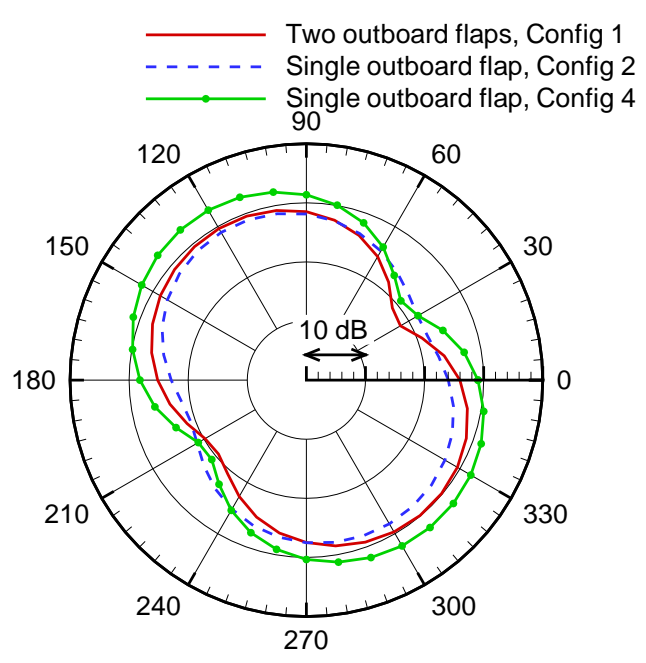

(b) OASPL.

Figure 13. Comparison of the three configurations with SPL and OASPL of the noise, which is induced by the boundary layers on the outboard flap group. 


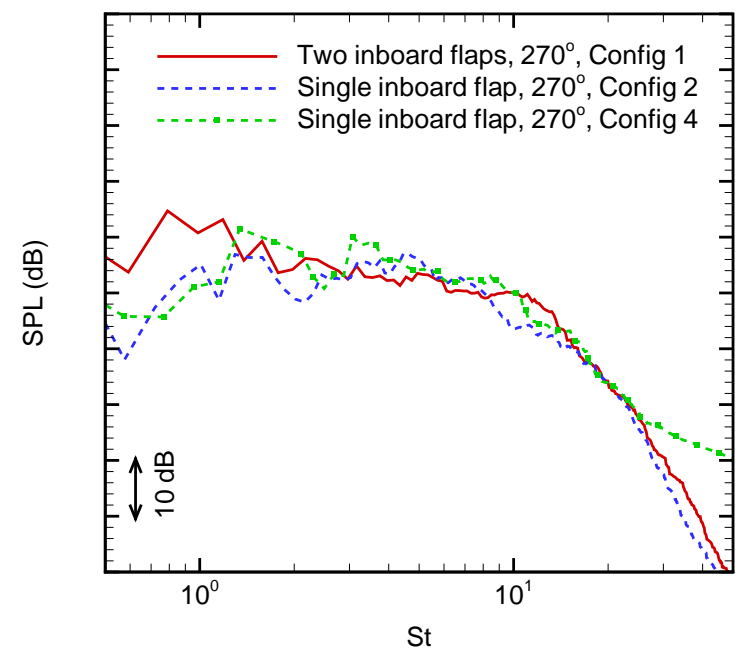

(a) SPL at $270^{\circ}$.

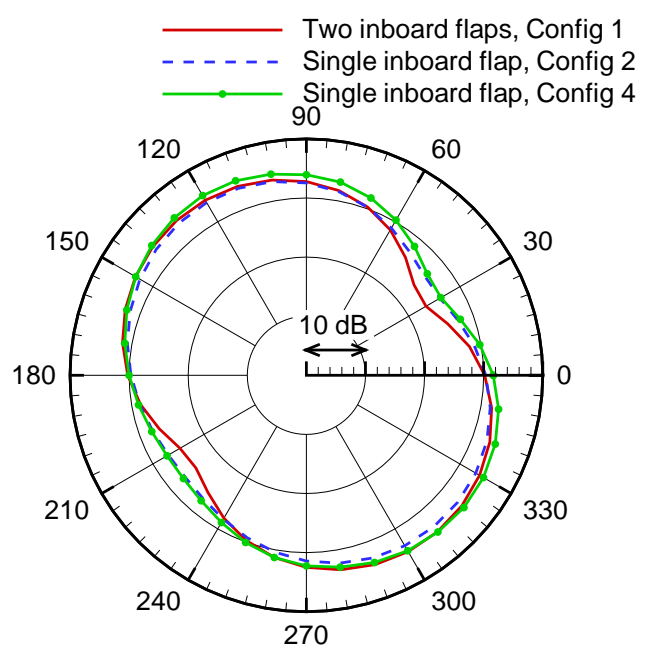

(b) OASPL

Figure 14. Comparison of the three configurations with SPL and OASPL of the noise, which is induced by the boundary layers on the inboard flap group.

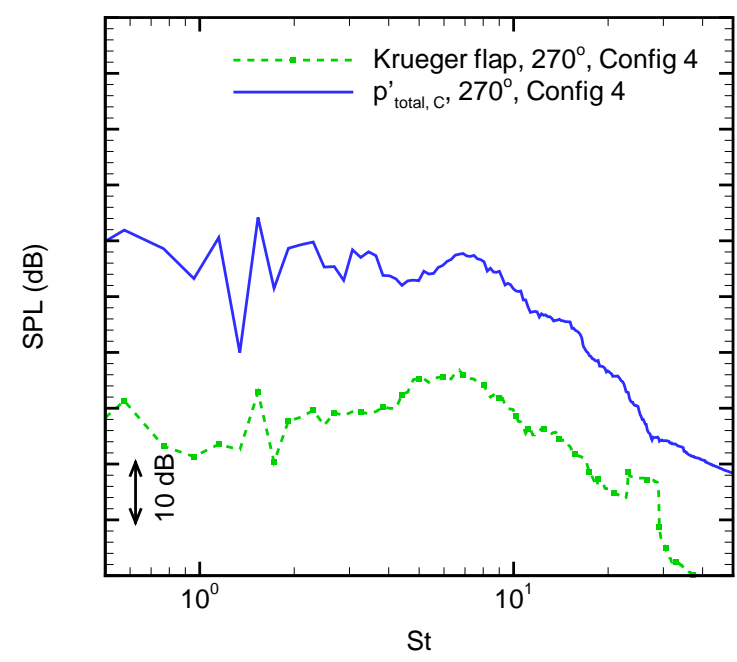

(a) SPL at $270^{\circ}$.

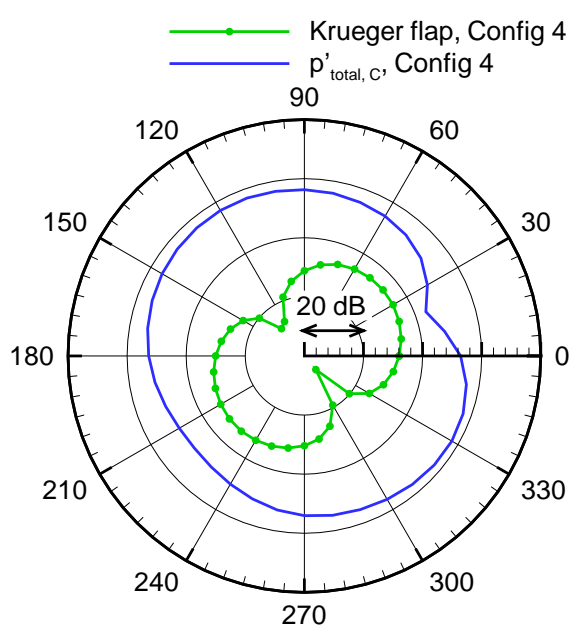

(b) OASPL

Figure 15. SPL and OASPL for the noise induced by the boundary layer of the Krueger flap surface are compared with those for the total noise $p_{t o t a l, C}^{\prime}$ by all surfaces. 
The noise induced by the boundary layers of the common configuration components is studied using the Curle method. The noise related to the wing surface of the last two configurations is reduced with almost equal levels compared with the first one. However, the cove regions of the last two configurations induce more noise instead of a reduction. For the outboard flap group, the noise from Config. 4 is highest. However, the inboard flap group is present as a common dominant noise source among all components. Moveover, it is observed that the Krueger flap of Config. 4 is an insignificant noise source in contrast to the other components.

\section{Acknowledgments}

The work has partly been supported by the Clean Sky Joint Undertaking (CSJU) under contract No. CSGA-2009-255714. The industrial monitor of this project is Doctor Michele Averardo at Alenia Aeronautica.

\section{References}

${ }^{1}$ Farassat, F. and Myers, M. K., "Extension of Kirchhoffs Formula to Radiation from Moving Surfaces," Journal of Sound and Vibration, Vol. 123, No. 3, 1988, pp. 451-461.

${ }^{2}$ Lyrintzis, A. S., "Review: the Use of Kirchhoff Method in Computational Aeroacoustics," ASME Journal of Fluids Engineering, Vol. 116, No. 4, 1994, pp. 665-676.

${ }^{3}$ Farassat, F., "Acoustic Radiation from Rotating Blades - the Kirchhoff Method in Aeroacoustics," Journal of Sound and Vibration, Vol. 239, No. 4, 2001, pp. 785-800.

${ }^{4}$ Lockard, D. P. and Choudhari, M. M., "Noise Radiation from a Leading-Edge Slat," 15th AIAA/CEAS Aeroacoustics Conference, AIAA 2009-3101, 2009.

${ }^{5}$ Manoha, E., Redonnet, S., Delahay, C., Sagaut, P., Mary, I., Khelil, S. B., and Guillen, P., "Numerical Prediction of the Unsteady Flow and Radiated Noise from a 3D Lifting Airfoil," IRTO AVT Symposium on Ageing Mechanisms and Control: Part A - Developments in Computational Aero- and Hydro-Acoustics, Manchester, UK, 2001.

${ }^{6}$ di Francescantonio, P., "A New Kirchhoff Formulation for Transonic Rotor Noise," Journal of Sound and Vibration, Vol. 202, No. 4, 1997, pp. 491-509.

${ }^{7}$ Brentner, K. S. and Farassat, F., "An Analytical Comparison of the Acoustic Analogy and Kirchhoff Formulation for Moving Surfaces," AIAA Journal, Vol. 36, No. 8, 1998, pp. 1379-1386.

${ }^{8}$ Brentner, K. S. and Farassat, F., "Modeling Aerodynamically Generated Sound of Helicopter Rotors," Progress in Aerospace Sciences, Vol. 39, 2003, pp. 83-120.

${ }^{9}$ Prieur, J. and Rahier, G., "Aeroacoustic integral methods, formulation and efficient numerical implementation," Aerospace Science and Technology, Vol. 5, 2001, pp. 457-468.

${ }^{10}$ Lyrintzis, A. S. and Uzun, A., "Integral Techniques for Jet Aeroacoustics Calculations," 7th AIAA/CEAS Aeroacoustics Conference, Maastricht, Netherlands, AIAA 2001-2253, 2001.

${ }^{11}$ Pan, F. L., Uzun, A., and Lyrintzis, A. S., "Surface Integral Methods in Jet Aeroacoustics: Refraction Corrections," Journal of Aircraft, Vol. 45, No. 2, 2008, pp. 381-387.

${ }^{12}$ Ewert, R., Dierke, J., Mühlbauer, B., Neifeld, A., Appel, C., Siefert, M., and Kornow, O., "CAA Broadband Noise Prediction for Aeroacousitc Design," IUTAM Symposium on Computational Aero-Acoustics for Aircraft Noise prediction, 2010.

${ }^{13}$ Ewert, R. and Schröder, W., "On the Simulation of Trailing Edge Noise with a Hybrid LES/APE Method," Journal of Sound and Vibration, Vol. 270, 2004, pp. 509-524.

${ }^{14}$ Curle, N., "The Influence of Solid Boundaries Upon Aerodynamic Sound," Proc. Roy. Soc., Vol. A 231, 1955, pp. 505-514.

${ }^{15}$ Goldstein, M. E., editor, Aeroacoustics, McGraw-Hill Book Company, New York, 1976.

${ }^{16}$ Wagner, C., Hüttl, T., and Sagaut, P., editors, Large-Eddy Simulation for Acoustics, Cambridge University, 2007.

${ }^{17}$ Yao, H. D., Davidson, L., Eriksson, L. E., Grundestam, O., Peng, S. H., and Eliasson, P. E., "Surface Integral Analogy Approaches to Computing Noise Generated by a 3D High-Lift Wing Configuration," 50th AIAA Aerospace Sciences Meeting, AIAA 2012-386, 2012.

${ }^{18}$ Williams, J. E. F. and Hawkins, D. L., "Sound Generation by Turbulence and Surfaces in Arbitrary Motion," Philos. Trans. Roy. Soc., Vol. A 264, No. 1151, 1969, pp. 321-342.

${ }^{19}$ Eliasson, P. E., Grundestam, O., Peng, S. H., Yao, H. D., Davidson, L., and Eriksson, L. E., "Assessment of High-lift Concepts for a Regional Aircraft in the ALONOCO Project," 50th AIAA Aerospace Sciences Meeting, AIAA 2012-277, 2012. 